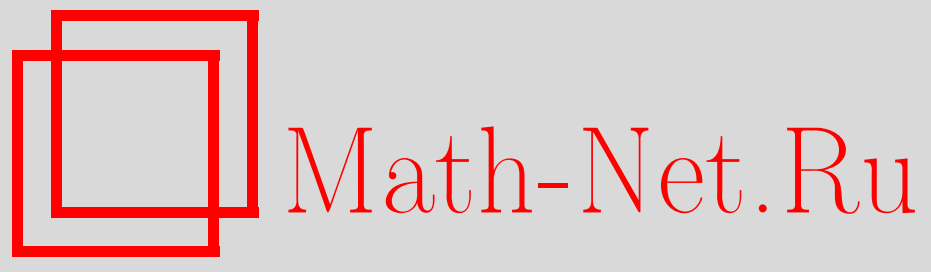

А. В. Сильниченко, О сходимости порядкосохраняющих слабых жадных алгоритмов, Матем. заметки, 2008, том 84, выпуск 5, 795-800

DOI: https://doi.org/10.4213/mzm6365

Использование Общероссийского математического портала Math-Net.Ru подразумевает, что вы прочитали и согласны с пользовательским соглашением http://www . mathnet.ru/rus/agreement

Параметры загрузки:

IP: 3.80 .181 .102

26 апреля 2023 г., 17:29:00

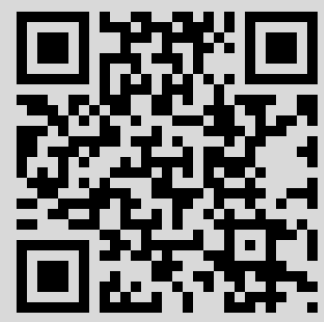




\section{О сходимости порядкосохраняющих слабых жадных алгоритмов}

\section{А. В. Сильниченко}

1. Введение и формулировки основных результатов. В статье речь пойдет о сходимости порядкосохраняющих слабых жадных алгоритмов (далее ПСЖА) в различных пространствах. Во второй части мы получим критерий сходимости ПСЖА в произвольном нормированном пространстве. В третьей и четвертой частях мы приведем два следствия из общего критерия: первое будет касаться сходимости ПСЖА в $L_{p}$ по специальным системам функций, а второе - геометрической интерпретации критерия в гильбертовом пространстве.

Пусть $X$ - квазинормированное пространство. (Вместо неравенства треугольника для любых $x, y \in X$ имеем $\|x+y\| \leqslant C(\|x\|+\|y\|)$, где $C$ - абсолютная константа, например, $L_{p}$ с квазинормой $\|f\|_{p}=\left(\int|f(x)|^{p} d x\right)^{1 / p}$ при $0<p<1$.)

Пусть $\left\{L_{k}\right\}$ - последовательность линейных подпространств из $X,\left\{\alpha_{k}\right\}$ - последовательность неотрицательных чисел. Определим ПСЖА для любого элемента $f \in X$ с помощью следующей индуктивной процедуры. Пусть $f_{0}(f)=0, r_{0}(f)=f, k_{0}(f)=0$. Если определены $f_{n}(f), r_{n}(f)$ и $k_{n}(f)$, то выберем $k_{n+1}=k_{n+1}(f)>k_{n}(f)$ и элемент $\varphi_{k_{n+1}} \in L_{k_{n+1}}$ так, чтобы

$$
\left\|r_{n}(f)-\varphi_{k_{n+1}}\right\| \leqslant \inf _{\varphi \in L_{k}, k>k_{n}}\left\|r_{n}(f)-\varphi\right\|+\alpha_{n} .
$$

Далее положим $f_{n+1}(f)=f_{n}(f)+\varphi_{k_{n+1}}$ и $r_{n+1}(f)=f-f_{n+1}(f)$.

В отличие от обычных жадных алгоритмов, мы хотим при приближении сохранять заданный порядок элементов системы (подробнее о жадных алгоритмах можно прочитать в [1]).

Рассмотрим следующую задачу: при каких условиях на систему подпространств $\left\{L_{k}\right\}$ ПСЖА по этой системе сходится для любой последовательности $\left\{\alpha_{k}\right\}_{k=0}^{\infty}$, стремящейся к 0 .

Во второй части статьи мы докажем критерий сходимости ПСЖА в квазинормированном пространстве с равномерно непрерывной квазинормой. А именно, для любых $\varepsilon>0, R>0$ существует $\delta>0$ такое, что для любых векторов $x, \beta,\|\beta\|<\delta,\|x\|<R$, выполнено неравенство $\|x+\beta\| \leqslant\|x\|+\varepsilon$. Например, квазинорма в пространстве $L_{p}$ при любом $p>0$ равномерно непрерывна.

Теорема 1. Предположим, что $X$ - пространство с равномерно непрерывной квазинормой. ПСЖА по системе $\left\{L_{k}\right\}$ сходится для любой последовательности $\left\{\alpha_{k}\right\}_{k=0}^{\infty}$, $\alpha_{k} \rightarrow 0$ при $k \rightarrow \infty$ тогда и только тогда, когда существует $\sigma<1$ такое, что для любых $f \in X$ и $N$ существуют $n>N u \varphi \in L_{n}$ такие, ито

$$
\|f-\varphi\| \leqslant \sigma\|f\| .
$$

В третьей части статьи будет рассмотрена задача о системах представления в $L_{p}$, образованных сжатиями и сдвигами фиксированной функции. Система функций $\left\{\varphi_{n}\right\}_{n=0}^{\infty}$ называется системой представления в $X$, если для любого элемента $f \in X$ существует последовательность чисел $\left\{a_{n}\right\}_{n=0}^{\infty}$ такая, что $f=\sum_{n=0}^{\infty} a_{n} \varphi_{n}$ (при этом система $\left\{\varphi_{n}\right\}$ может не быть базисом, последовательность может быть не единственной). Пусть $\varphi \in$ $L_{p}(\mathbb{R}), \operatorname{supp} \varphi \subset[0,1]$. Рассмотрим систему двоичных сжатий сдвигов $\varphi_{k, i}(x)=\varphi\left(2^{k} x-i\right)$, $k \in \mathbb{Z}, k \geqslant 0, i=0, \ldots, 2^{k}-1$. Возникает задача: при каких условиях система $\left\{\varphi_{k, i}\right\}$ является системой представления в $L_{p}$ ?

(C) А. В. Сильниченко, 2008 
В [2] доказаны следующие утверждения:

1) при $p \geqslant 1, \int_{0}^{1} \varphi(x) d x \neq 0, \varphi \in L_{p}$, система $\left\{\varphi_{k, i}\right\}$ является системой представления в $L_{p}$;

2) при $p<1, \varphi \neq 0, \varphi \in L_{2}$ система $\left\{\varphi_{k, i}\right\}$ является системой представления в $L_{p}$.

Позже Ф. П. Назаров показал (устное сообщение), что для любого $p<1$ существует число $q(p)<2$ такое, что при $\varphi \in L_{q}$ система $\left\{\varphi_{k, i}\right\}$ является системой представления в $L_{p}$.

Используя теорему 1 , мы уточняем данный результат и получаем верхнюю границу для $q(p)$.

Пусть $L_{k}$ - подпространства, натянутые на вектора $\left\{\varphi_{k, i}\right\}$, где $k$ - фиксировано, а $i=$ $0, \ldots, 2^{k}-1$.

Теорема 2. Пусть $p \in(0,1)$ и $\varphi$-действительнозначная функиия с носителем на отрезке $[0,1], \varphi \neq 0$. Если $\varphi \in L_{p+1}[0,1]$, то для любой последовательности $\left\{\alpha_{k}\right\}_{k=0}^{\infty}$, $\alpha_{k} \rightarrow 0$ при $k \rightarrow \infty$, ПСЖА по системе подпространств $L_{k}, k \geqslant 0$, сходится для любой функиии $f \in L_{p}[0,1]$.

Также будут получены условия, достаточные для сходимости ПСЖА почти всюду.

Теорема 3. В условиях теоремы 2 ПСЖА сходится почти всюду на [0,1], если $\sum_{i=0}^{\infty} \alpha_{n}<\infty$.

В четвертой части работы будут в некоторых частных случаях получены геометрические формулировки критерия сходимости ПСЖА. В случае гильбертова пространства из теоремы 1 удается получить критерий сходимости ПСЖА в геометрических терминах. Пусть $H$ - гильбертово пространство. Точку $\psi \in H$ будем называть пределом в слабом смысле для последовательности $\left\{\varphi_{n}\right\}$, если для любого $g \in H$ выполнено: $\left(g, \varphi_{n}\right) \rightarrow(g, \psi)$ при $n \rightarrow \infty$. Пусть $B$ - множество точек $\psi$, для которых существует последовательность $\left\{\varphi_{n}\right\}, \varphi_{n} \in L_{n},\left\|\varphi_{n}\right\|=1$, слабо сходящаяся к $\psi$. Пусть $A$ - замыкание выпуклой оболочки множества $B$.

ТЕОРемА 4. ПСЖА по системе $\left\{L_{n}\right\}$ сходится для любой последовательности $\left\{\alpha_{k}\right\}_{k=0}^{\infty}, \alpha_{k} \rightarrow 0$ при $k \rightarrow \infty$ тогда и толъко тогда, когда внутренность множества $A$ не пуста.

2. Доказательство теоремы 1. Начнем со следующей простой леммы.

ЛЕмма 1. Пусть для норм остатков верно следующее неравенство:

$$
\left\|r_{n}\right\| \leqslant \sigma^{n}\|f\|+\sum_{i=1}^{n} \sigma^{n-i} \alpha_{i} .
$$

Тогда $\left\|r_{n}\right\| \rightarrow 0$ при $n \rightarrow \infty$.

ДоказАтельство. Докажем, что $\left\|r_{n}\right\| \rightarrow 0$ при $n \rightarrow \infty$. Возьмем произвольное $\varepsilon>0$. Существует $N$ такое, что $\sigma^{N}\|f\|<\varepsilon$ и для любого $n>N$ выполнено $\left|\alpha_{n}\right|<\varepsilon$. Существует $K \geqslant 0$ такое, что $\sum_{i=1}^{N} \sigma^{K+N-i} \alpha_{i}<\varepsilon$. Тогда для любого $n>N+K$ имеем

$$
\begin{aligned}
\left\|r_{n}\right\| & \leqslant \sigma^{n}\|f\|+\sum_{i=1}^{n} \sigma^{n-i} \alpha_{i} \\
& \leqslant \varepsilon+\sum_{i=1}^{N} \sigma^{K+N-i} \alpha_{i}+\sum_{i=N+1}^{n} \sigma^{n-i} \alpha_{i} \leqslant \varepsilon+\varepsilon+\frac{1}{1-\sigma} \varepsilon=\left(2+\frac{1}{1-\sigma}\right) \varepsilon .
\end{aligned}
$$

Лемма доказана. 
ДокАЗАТЕЛЬСтво теОРемы 1. Утверждение "тогда" вытекает из леммы 1. Если выполнено условие (1), то для норм остатка на каждом шаге алгоритма верна оценка (2) и, как следствие, $\left\|r_{n}\right\| \rightarrow 0$ при $n \rightarrow \infty$.

В обратную сторону будем доказывать теорему методом от противного. Пусть ПСЖА сходится для любого элемента $f \in X$, но условие (1) не выполнено. Тогда для любого $\sigma<1$ существуют $f \in X$ и $N$ такие, что для любых $n>N$ и $\varphi \in L_{n}$ справедливо неравенство $\|f-\varphi\|>\sigma\|f\|$. В частности, существуют последовательность $\left\{f_{k}\right\},\left\|f_{k}\right\|=1$ и числа $N_{k}$ такие, что для любых $n>N_{k}$ и $\varphi \in L_{n}$ выполнено $\left\|f_{k}-\varphi\right\|>(1-1 / k)$. Ошибкой $n$-го шага ПСЖА будем называть величину

$$
E_{n}=\left\|r_{n}-\varphi_{n+1}\right\|-\inf _{\varphi \in L_{k}, k>n}\left\|r_{n}-\varphi\right\|
$$

Построим реализацию ПСЖА, которая не будет сходиться. Воспользуемся методом математической индукции. Возьмем $r_{0}=f_{1}$ и последовательность $\left\{\delta_{k}\right\}$ такую, что $\delta_{k}<1 / k$, $k \in \mathbb{N}$, причем для $x, x+\beta,\|x\|<2,\|x+\beta\|<2,\|\beta\|<\delta_{k}$, выполнено $\|x+\beta\| \leqslant\|x\|+1 / k$. Пусть после $n_{k, 0}$ шагов алгоритма имеем $r_{n_{k, 0}}=f_{k}+\gamma_{k, 0}$, где $\left\|\gamma_{k, 0}\right\|<\delta_{k} / 2$. Докажем, что от $f_{k}$ можно перейти к $f_{k+1}$ за конечное число шагов алгоритма, причем ошибка на каждом шаге не превосходит $4 / k$. Так как ПСЖА сходится для любого элемента из $X$, для любых $\varepsilon>0$ и последовательности $\left\{\alpha_{i}\right\}, \alpha_{i} \rightarrow 0$, через конечное число шагов получим $\left\|r_{i}\right\|<\varepsilon$. Взяв числа $\alpha_{i}=1 /(i+k)$, получим $r_{n_{k, 1}}=\gamma_{k, 1},\left\|r_{n_{k, 1}}\right\|<\delta_{k} / 2$. Рассмотрим $r_{n_{k, 1}}=(1 / 2) \delta_{k} f_{k+1}+\gamma_{k, 1}-(1 / 2) \delta_{k} f_{k+1}$. Заметим, что $\left\|\gamma_{k, 1}-(1 / 2) \delta_{k} f_{k+1}\right\|<2 / k$. Приблизим с помощью ПСЖА по системе $\left\{L_{n}\right\}_{n>n_{k, 1}}$ вектор $\gamma_{k, 1}-(1 / 2) \delta_{k} f_{k+1}$. Возьмем коэффициенты приближения $\alpha_{j}=2^{-(j+k)}$. Тогда

$$
\left\|r_{j}\left(\gamma_{k, 1}-\frac{1}{2} \delta_{k} f_{k+1}\right)\right\| \leqslant\left\|\gamma_{k, 1}-\frac{1}{2} \delta_{k} f_{k+1}\right\|+\sum_{i=1}^{j} \alpha_{i} \leqslant \frac{3}{k} .
$$

Так как ПСЖА сходится, существует $J$ такое, что $\left\|r_{J}\right\|<\delta_{k}$.

Вычислим максимальную ошибку ПСЖА, если для приближения $r_{n_{k, 1}}$ брать последовательно те же приближающие элементы, что и для $\gamma_{k, 1}-(1 / 2) \delta_{k} f_{k+1}$. Так как $\left\|(1 / 2) \delta_{k} f_{k+1}\right\|<\delta_{k}$, получим $\left\|r_{n_{k, 1+j}}\right\| \leqslant\left\|r_{j}\left(\gamma_{k, 1}-(1 / 2) \delta_{k} f_{k+1}\right)+1 / k\right\|<4 / k$. При этом суммарная ошибка не превзойдет $4 / k$, так как ничего лучше нулевого вектора получить нельзя. Таким образом, на $n_{k, 2}$ шаге ПСЖА имеем $r_{n_{k, 2}}=(1 / k) f_{k+1}+\gamma_{k, 2}$. Воспользуемся методом математической индукции по второму индексу. Пусть построена реализация ПСЖА такая, что $r_{n_{k, i}}=(i-1) \delta_{k} f_{k+1}+\gamma_{k, i},\left\|\gamma_{k, i}\right\|<\delta_{k}$. Покажем, что от $r_{n_{k, i}}$ можно с помощью некоторого числа шагов перейти к $r_{n_{k, i+1}}$, и ошибка на каждом шаге не превысит $7 / k$. Рассмотрим $r_{n_{k_{i}}}=i \delta_{k} f_{k+1}+\gamma_{k, i}-\delta_{k} f_{k+1}$. Приблизим $\gamma_{k, i}-\delta_{k} f_{k+1}$ с помощью ПСЖА по системе $\left\{L_{n}\right\}_{n>n_{k, i}}$ с $\alpha_{j}=2^{-(k+j)}$. Так как ПСЖА сходится, существует $J$ такое, что $\left\|r_{J}\left(\gamma_{k, i}-\delta_{k} f_{k+1}\right)\right\|<\delta_{k}$. Вычислим максимальную ошибку ПСЖА для элемента $r_{n_{k, i}}$, если в качестве приближающих векторов брать те же векторы, что и для $\gamma_{k, i}-\delta_{k} f_{k+1}$. Из непрерывности квазинормы и выбора $f_{k+1}$ имеем

$$
i \delta_{k}\left(1-\frac{1}{k+1}\right)\left\|f_{k+1}\right\| \leqslant\left\|i \delta_{k} f_{k+1}-\varphi\right\| \leqslant \frac{1}{k}+\left\|i \delta_{k} f_{k+1}+\gamma_{k, i}-\varphi\right\| .
$$

Следовательно, $\left\|(i / k) f_{k+1}+\gamma_{i}-\varphi\right\| \geqslant(i-2) / k$. Значит, нельзя уменьшить норму остатка за один шаг больше, чем на $2 / k$. Тогда суммарная ошибка не превосходит $2 / k+\left\|\gamma_{k, i}\right\|+$ $1 / k<4 / k$, где последнее слагаемое отвечает за точность приближения $\gamma_{k, i}$. Получили реализацию ПСЖА, для которой $r_{n_{k, 0}}=f_{k}+\gamma_{k, 0}$, где $\left\|\gamma_{k, 0}\right\|<\delta_{k}$ для всех $k$.

Полученная реализация не сходится, так как норма остатка не стремится к 0. Данное противоречие доказывает теорему. 
СледСтвиЕ 1. В условиях теоремы 1 для скорости сходимости ПСЖА верна оченка

$$
\left\|r_{n}\right\| \leqslant \sigma^{n}\|f\|+\sum_{i=1}^{n} \sigma^{n-i} \alpha_{i} .
$$

3. ПСЖА в функциональных пространствах. Перед доказательством теоремы 2 сделаем несколько замечаний.

СледствиЕ 2. В условиях теоремы $2\left\{\varphi_{k, i}\right\}$ является системой представления в $L_{p}$.

ДокАзАтельство. Так как носители функций $\left\{\varphi_{k, i}\right\}$ не пересекаются при любом фиксированном $k$ и $i=0, \ldots, 2^{k}-1$, из сходимости ПСЖА следует сходимость порожденного им ряда.

ЗАмечание 1 . Теорема 1 не улучшаема в данных терминах. А именно, для любого $q<p+1$ существуют функции $\varphi \in L_{q}$ и $f \in L_{p}$ такие, что ПСЖА для $f$ по системе двоичных сжатий и целых сдвигов функции $\varphi$ не сходится к $f$ ни для какой последовательности $\left\{\alpha_{k}\right\}$.

ПримеР 1. Возьмем $f(x)=1$ для любого $x, \varphi(x)=x^{-1 /(p+1)}, x \in[0,1 / 2]$ и $\varphi(x)=$ $-(1-x)^{-1 /(p+1)}, x \in[1 / 2,1]$. В работе [3] показано, что $\|1-\lambda \varphi\|_{p} \geqslant 1$ для любого $\lambda$. Следовательно, на каждом шаге алгоритма может быть взято нулевое приближение и алгоритм сойдется к тождественному нулю.

Доказательству теоремы предпошлем лемму.

Лемма 2. В условиях теоремы 1 существует $\lambda$ такое, что

$$
\|1-\lambda \varphi\|_{p} \geqslant 1 .
$$

ДокАЗАтЕЛЬСтво. Рассмотрим двойной интеграл

$$
I=\int_{0}^{1}\left(\int_{0}^{+\infty}\left(|1+\lambda \varphi(x)|^{p}+|1-\lambda \varphi(x)|^{p}-2\right) \lambda^{-p-2} d \lambda\right) d x .
$$

Очевидно, что внутренний интеграл сходится абсолютно. Заметим, что

$$
I=\int_{0}^{1}\left(\int_{0}^{+\infty}\left(|1+\lambda| \varphi(x)\left\|^{p}+|1-\lambda| \varphi(x)\right\|^{p}-2\right) \lambda^{-p-2} d \lambda\right) d x .
$$

Сделав замену $\mu=\lambda|\varphi(x)|$ (так как в точках $x$, для которых $\varphi(x)=0$, исходный и полученный после замены интегралы равны 0 , такая замена возможна), получим

$$
I=\int_{0}^{+\infty}\left(|1+\mu|^{p}+|1-\mu|^{p}-2\right) \mu^{-p-2} d \mu \int_{0}^{1}|\varphi(x)|^{p+1} d x .
$$

Докажем, что это произведение равно 0. Сделав замену $t=1 / \mu$ в первом интеграле произведения получим

$$
\begin{aligned}
\int_{0}^{+\infty} & \left(|1+t|^{p}+|1-t|^{p}-2 t^{p}\right) d t \\
& =\lim _{M \rightarrow \infty} \int_{0}^{M}\left(|1+t|^{p}+|1-t|^{p}-2 t^{p}\right) d t \\
& =\lim _{M \rightarrow \infty} \frac{1}{p+1}\left((1+M)^{p+1}+(M-1)^{p+1}-2 M^{p+1}-2\right) \\
& =\lim _{M \rightarrow \infty} \frac{1}{p+1} M^{p+1}\left((1+1 / M)^{p+1}+(1-1 / M)^{p+1}-2\right)=0 .
\end{aligned}
$$


Переписав интеграл (3) в виде

$$
0=\int_{0}^{+\infty} \lambda^{-p-2} d \lambda\left(\left(\int_{0}^{1}|1+\lambda \varphi(x)|^{p} d x-1\right)+\left(\int_{0}^{1}|1-\lambda \varphi(x)|^{p} d x-1\right)\right),
$$

получим: или существует $\lambda$, при котором $\int_{0}^{1}|1+\lambda \varphi(x)|^{p} d x<1$, или при любом $\lambda$ имеем $\int_{0}^{1}|1+\lambda \varphi(x)|^{p} d x=1$. Но второй случай не может выполняться, так как $\int_{0}^{1} \mid 1+$ $\left.\lambda \varphi(x)\right|^{p} d x>1$ при достаточно больших $\lambda$. Лемма доказана.

Лемма 3. Пусть функиия $\varphi(x) \in L_{p}, \varphi \neq 0$, удовлетворяет условиям леммы 2. Тогда для любой $f \in L_{p}$ и для любого $N$ существуют $n>N$ и набор чисел $\left\{c_{n, i}\right\}$ такие, что $\left\|f-\sum_{i=0}^{2^{n}-1} c_{n, i} \varphi_{n, i}\right\|_{p}<\sigma\|f\|_{p}$, әде $\sigma<1$.

ДокАЗАТЕЛЬСтво леммы 3 содержится в работе [2].

Из леммы 3 следует выполнение условий теоремы 1. Следовательно, ПСЖА сходится. Теорема 2 доказана.

ДОКАЗАТЕЛЬСТВО тЕОРЕМЫ 3. Воспользуемся следующей простой леммой.

Лемма 4. Пусть $\Omega$ - пространство с мерой $\mu, \mu(\Omega)<\infty,\left\{f_{n}\right\}-$ последовательность функций из $L_{p}(\Omega)$, сходящаяся к некоторой функции $f$. Если $\sum_{n=1}^{\infty}\left\|f-f_{n}\right\|_{p}<\infty$, то последовательность $f_{n}$ сходится $\kappa$ почти всюду на $\Omega$.

ЗАмечАниЕ 2. Условие леммы 4 не может быть усилено в данных терминах, а именно, для любой последовательности $r_{n} \rightarrow 0, \sum_{n=1}^{\infty} r_{n}=\infty$, существует последовательность функций $f_{n}$, сходящаяся к функции $f$ по норме $L_{p},\left\|f-f_{n}\right\|_{p} \leqslant r_{n}$, и расходящаяся всюду на $\Omega$.

Пример 2. Возьмем $\Omega=[0,1), f_{n}=1$ при $x \in\left[\max \left(0,\left\{\sum_{k=1}^{n} r_{k}\right\}-r_{n}\right),\left\{\sum_{k=1}^{n} r_{k}\right\}\right]$, где $\{\cdot\}$ - дробная часть числа, и $f_{n}(x)=0$ при других $x \in[0,1)$.

ДоказАтельство ЛЕммы 4. Обозначим $r_{n}=\left\|f-f_{n}\right\|_{p}$. Пусть $\varepsilon \in(0,1)$. Рассмотрим множества $E_{n}=\left\{x:\left|f(x)-f_{n}(x)\right|>\varepsilon\right\}$. Очевидно, что $\mu\left(E_{n}\right) \leqslant \varepsilon^{-p} r_{n}$. Так как $\sum_{n=1}^{\infty} r_{n}<\infty$, то $\sum_{n=1}^{\infty} \mu\left(E_{n}\right)<\infty$. Следовательно, для любого $\delta>0$ существует $N$ и множество $Q, \mu(Q)<\delta$, такие, что для любого $x \in \Omega \backslash Q$ и $n>N$ верно $\mid f(x)-$ $f_{n}(x) \mid<\varepsilon$. Возьмем последовательность $\varepsilon_{n}=1 / n$ и найдем по ней множества $Q_{n}$, $\mu\left(Q_{n}\right)<\delta / 2^{n}$. Рассмотрим множество $Q=\bigcup_{n=1}^{\infty} Q_{n}$. Его мера меньше $\delta$, и для любого $x \in \Omega \backslash Q$ последовательность $\left\{f_{n}(x)\right\}$ сходится к $f(x)$. Из условия произвольности $\delta$ получим утверждение леммы.

Утверждение теоремы 3, очевидно, следует из леммы 4 и оценки (2).

4. Критерий сходимости ПСЖА в гильбертовом пространстве. Доказательству теоремы 4 предпошлем лемму.

ЛЕмма 5. ПСЖА по системе $\left\{L_{k}\right\}$ сходится для любой последовательности $\left\{\alpha_{k}\right\}$, $\alpha_{k} \rightarrow 0$ при $k \rightarrow \infty$, для любого $f \in H$ тогда и только тогда, когда

$$
\inf _{f \in H\|f\|=1} \sup _{\psi \in A}|(f, \psi)|>0 .
$$

ДокАЗАТЕЛЬСтво. Пусть

$$
q=\inf _{f \in H\|f\|=1} \sup _{\psi \in A}|(f, \psi)|>0 .
$$

Тогда

$$
q=\inf _{f \in H\|f\|=1} \varlimsup_{k \rightarrow \infty} \sup _{\varphi \in L_{k}}|(f, \varphi)|
$$

и, значит, условие $(1)$ выполнено с $\sigma=\sqrt{1-q^{2} / 4}$, и по теореме 1 ПСЖА сходится. 
Докажем лемму в другую сторону. Так как ПСЖА сходится, по теореме 1 выполнено условие (1). Найдется $q>0$ такое, что для любых $f \in H$ и $N$ существуют $n>N$ и $\varphi \in L_{n}$ такие, что $|(f, \varphi)|>q$. Следовательно, существует подпоследовательность $\left\{\varphi_{n_{k}}\right\}$, $\varphi_{n_{k}} \in L_{n_{k}}$, для которой выполнено $\left|\left(f, \varphi_{n_{k}}\right)\right|>q$. Пусть $\psi$ - слабая предельная точка последовательности $\left\{\varphi_{n_{k}}\right\}$. Тогда $|(f, \psi)|>q$, и в силу произвольности $f$ выполнено условие (4). Лемма доказана.

Перейдем непосредственно к доказательству теоремы 4. Если внутренность $A$ не пуста, то, поскольку множество $A$ симметрично относительно 0 , в нем содержится шар с центром в нуле и некоторым радиусом $q>0$. Тогда для множества $A$ выполнено условие (4) и по лемме 5 ПСЖА сходится.

Пусть внутренность множества $A$ пуста. Тогда для любого $n=1,2, \ldots$ найдется элемент $g_{n} \in H$ такой, что $\left\|g_{n}\right\|<1 / n$ и $g_{n}$ не принадлежит $A$. По теореме отделимости существует элемент $f_{n} \in H$ такой, что $\left\|f_{n}\right\|=1$ и $\left(f_{n}, g_{n}\right)>\sup _{\psi \in A}\left(f_{n}, \psi\right)$. Учитывая симметричность множества $A$, выводим отсюда, что $\sup _{\psi \in A}\left|\left(f_{n}, \psi\right)\right|<1 / n$. Следовательно, условие (4) не выполнено и по лемме 4 ПСЖА не сходится. Теорема доказана.

\section{СПИСОК ЦИТИРОВАННОЙ ЛИТЕРАТУРЫ}

[1] V. N. Temlyakov, Foundations of Computational Mathematics (Santander, 2005), London Math. Soc. Lecture Note Ser., 331, Cambridge Univ. Press, Cambridge, 2006, 371-394. [2] V. I. Filippov, P. Oswald, J. Approx. Theory, 82:1 (1995), 15-29. [3] В. И. Иванов, Тезисы докладов Всероссийской конферениии "Современные проблемы математики, механики, информатики", ТулГУ, Тула, 2000, 33-34.

\section{А. В. Сильниченко}

Поступило

Российский университет дружбы народов 